\section{Pancreatic melanoma metastasis diagnosed by endoscopic ultrasound-guided SharkCore biopsy}

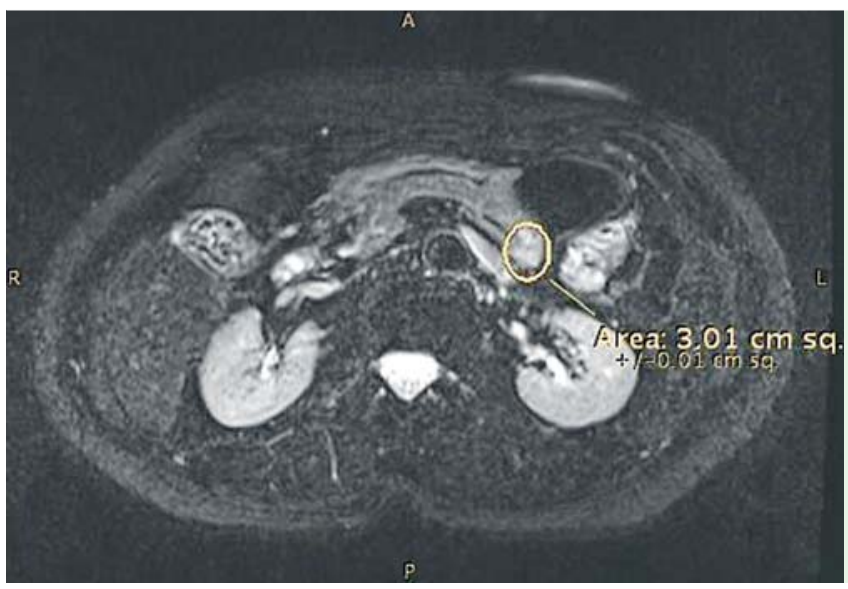

Fig. 1 T1-weighted contrast-enhanced magnetic resonance imaging (MRI) scan showing a $20 \times 17-\mathrm{mm}$ mass in the pancreatic tail, which appears to be both isointense and hyperintense (circled).

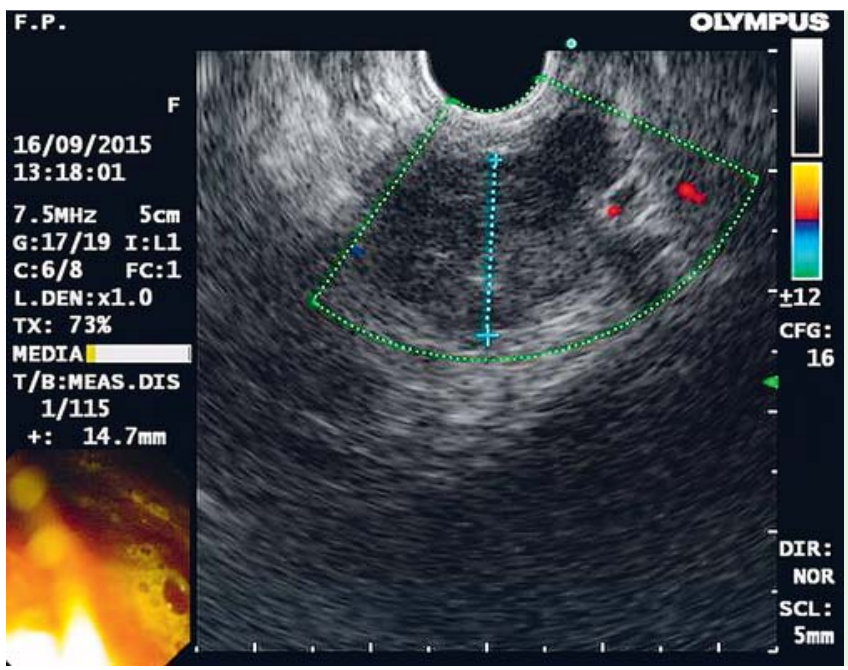

Fig. 2 Endoscopic ultrasound (EUS) image of the pancreas showing a $20-\mathrm{mm}$ round, hypoechoic mass with irregular borders in the tail of the pancreas.

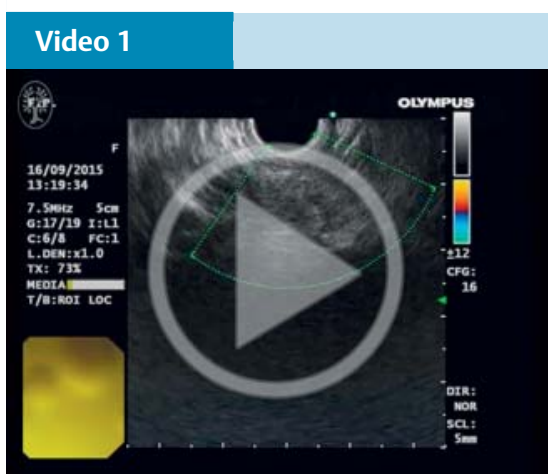

Endoscopic ultrasound (EUS) imaging of the mass in the pancreatic tail and transduodenal fine needle biopsies obtained with the SharkCore biopsy needle. by iodine-125 brachytherapy. At the time of treatment no metastatic lesions had been documented.

In May 2015, a magnetic resonance imaging (MRI) scan performed for clinical surveillance demonstrated a nodular liver consistent with metastasis. The patient underwent transarterial chemoembolization with irinotecan. A subsequent MRI scan showed a nodular lesion of the pancreatic tail $(20 \times 17 \mathrm{~mm})$, which was a mixture of isointense and hyperintense on T1-weighted images ( $\bullet$ Fig. $\mathbf{1}$ ), and was hypointense on T2-weighted images, compatible with either primary cancer or metastatic disease. The patient was asymptomatic, with no suspicious skin lesions and no palpable lymphadenopathy. Laboratory test results were within normal limits.

The patient was referred to our institution for EUS evaluation. The EUS (Olympus UCT 140 linear array echoendoscope) showed a 2-cm hypoechoic mass in the pancreatic tail ( Fig.2). Five transduodenal FNB passes (22-gauge SharkCore needle) into the mass resulted in multiple tissue samples (maximum diameter $0.08-0.1 \mathrm{~cm}$ ) (৫ Video 1). Histological examination showed malignant pigmented epithelioid lesions, which were immunohistochemically confirmed as metastatic melanoma ( $\bullet$ Fig.3). The patient underwent chemotherapy and has currently completed her induction cycles with fotemustine.

\section{Endoscopy_UCTN_Code_CCL_1AF_2AZ}

Competing interests: None

\section{Elena Nadal' ${ }^{1}$, Patrizia Burra², Claudia Mescoli ${ }^{3}$, Laura Albertoni ${ }^{3}$, Cosimo Sperti ${ }^{4}$, Giacomo Carlo Sturniolo $^{1}$, Alberto Fantin ${ }^{1}$}

${ }^{1}$ Gastroenterology Unit, Department of Surgery, Oncology and Gastroenterology, University Hospital of Padua, Padua, Italy ${ }^{2}$ Multivisceral Transplant Unit, Department of Surgery, Oncology and Gastroenterology, University Hospital of Padua, Padua, Italy

${ }^{3}$ Surgical Pathology \& Cytopathology Unit, Department of Medicine (DIMED), University Hospital of Padua, Padua, Italy 4 3rd Surgical Clinic, Department of Surgery, Oncology and Gastroenterology, University Hospital of Padua, Padua, Italy 


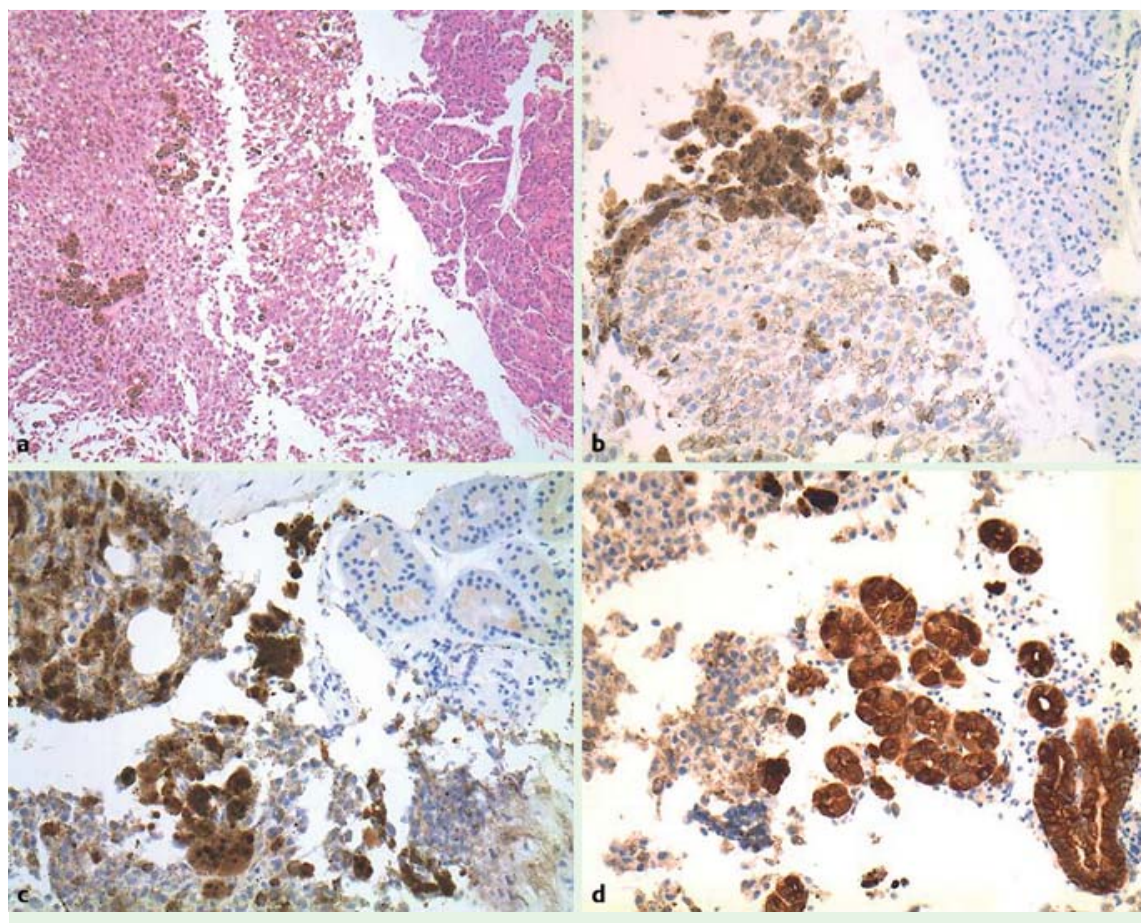

Fig. 3 Histopathology of the pancreatic tissue samples. a Hematoxylin and eosin (H\&E) staining shows normal pancreatic cells coexisting with a neoplastic (pigmented) cell population (original magnification $\times 10$ ). $\mathbf{b}-\mathbf{d}$ Immunohistochemical staining shows positivity with: $\mathbf{b}$ MNF 116 for epithelial cytokeratin (original magnification $\times 20$ ); c S100, a melanocytic marker (original magnification $\times 20$ ); d HMB-45, a melanocytic marker (original magnification $\times 20$ ).

\section{References}

1 Sperti C, Moletta L, Patanè G. Metastatic tumors to the pancreas: the role of surgery. World J Gastrointest Oncol 2014; 6: 381 392

2 Smith $A L$. Solid tumor metastases to the pancreas diagnosed by FNA: A single-institution experience and review of the literature. Cancer Cytopathology 2015; 123: $347-355$

\section{Bibliography}

DOI http://dx.doi.org/

10.1055/s-0042-109050

Endoscopy 2016; 48: E208-E209

(c) Georg Thieme Verlag KG

Stuttgart · New York

ISSN 0013-726X

\section{Corresponding author}

\section{Elena Nadal, MD}

Gastroenterology Unit

Department of Surgery, Oncology

and Gastroenterology

University Hospital of Padua

Via Nicolò Giustiniani, 2

35128 Padua

Italy

Fax: +39-049-8212887

elena.nadal@hotmail.it 